\title{
PERFIS PERFURADOS SUBMETIDOS À FLAMBAGEM TÉRMICA E MECÂNICA
}

\author{
Roberta da Silva Michaello ${ }^{1}$, Jorge Renato Soares Paes ${ }^{2}$, Liércio André Isoldi ${ }^{1}$, Rafael Gonzalez \\ Bastos $^{1}$, Elizaldo Domingues dos Santos ${ }^{1}$ \& Luiz, Alberto Oliveira Rocha ${ }^{3 *}$
}

\section{RESUMO}

MICHAELLO, R.S.; PAES, J.R.S.; ISOLDI, L.A.; BASTOS, R.G.; SANTOS, E.D.; ROCHA, L.A.O. Perfis perfurados submetidos à flambagem térmica e mecânica. Perspectivas Online: Exatas \& Engenharias, v. 8, n.20, p.116126,2018 .

Existem soluções analíticas para a determinação da carga crítica e da variação crítica de temperatura de colunas, desde que as mesmas não possuam perfurações. Se existirem o problema pode ser resolvido através de uma abordagem computacional. Então, neste trabalho, um modelo computacional verificado foi adotado para solucionar os problemas da flambagem mecânica e térmica. Para isso foi empregado o software ANSYS, que é baseado no Método dos Elementos Finitos (MEF). Um estudo de caso foi realizado, empregando o método Design Construtal para a determinação da influência na carga crítica e na variação crítica de temperatura em uma coluna de aço com perfurações. A forma dessas perfurações foram variadas, mantendo fixo o volume de material retirado da coluna. Os resultados obtidos mostraram que o método Design Construtal permite a determinação de geometrias otimizadas que conduzem a performances superiores dos componentes estruturais analisados. Estudos de flambagem mecânica e flambagem térmica considerando perfurações em perfis ou placas finas têm sido abordados em trabalhos isoladamente, sem a existência de uma correlação ou ponto comum entre eles. Então surgiu a motivação para realizar o presente trabalho, determinando, ainda que isoladamente, qual seria a geometria ótima dos furos, comum à flambagem mecânica e à flambagem térmica.

Palavras-chave: Flambagem; Design construtal; Modelagem computacional. 


\section{ABSTRACT}

There are analytical solutions for determining the critical load and the critical temperature variation of columns provided they do not have perforations. If they exist the problem can be solved through a computational approach. Then, in this work, a verified computational model was adopted to solve the problems of mechanical and thermal buckling. For this purpose, the ANSYS software was used, which is based on the Finite Element Method (MEF). A case study was carried out, using the Constructal Design method to determine the influence on the critical load and the critical temperature variation in a steel column with perforations. The shape of these perforations was varied, keeping fixed the volume of material removed from the column. The results showed that the Constructal Design method allows the determination of optimized geometries that lead to higher performances of the analyzed structural components. Studies of mechanical buckling and thermal buckling considering perforations in profiles or thin plates have been approached in works alone, without the existence of a correlation or common point between them. Then, the motivation to perform the present work was determined, even if alone, what would be the optimal geometry of the holes, common to mechanical buckling and thermal buckling.

Keywords: Buckling; Constructal design; Computational modeling.

\footnotetext{
${ }^{1}$ Universidade Federal do Rio Grande, FURG - Instituto de Oceanografia,Campus Carreiros, Avenida Itália, Km 8, s/n Carreiros, Rio Grande, RS, CEP: 96201-900,Brasil

${ }^{2}$ Escola de Guerra Naval, EGN - Av. Pasteur, 480 - Urca, Rio de Janeiro - RJ, CEP: 22290-240, Brasil

${ }^{3}$ Universidade Federal do Rio Grande do Sul, UFRGS - Av. Paulo Gama, 110, Farroupilha, Porto Alegre , RS, 90040060, Brasil.

(*)e-mail: luizrocha@mecanica.ufrgs.br

Data de chegada: 07/05/2017 Aceito para publicação: 29/05/2017
} 


\section{INTRODUÇÃO}

Elementos estruturais esbeltos, como as colunas metálicas, são amplamente empregados na construção de estruturas navais e offshore. Como exemplos, pode-se citar: a aplicação em estruturas de cascos de navios e a utilização em plataformas auto eleváveis.

Sempre que se projeta um elemento estrutural, é necessário que ele satisfaça requisitos específicos de resistência, rigidez e estabilidade. Em elementos estruturais sujeitos à compressão, sendo estes esbeltos e compridos, a carga poderá ser grande o suficiente para provocar uma deflexão ou uma oscilação lateral, fenômeno chamado de flambagem (Fig. 1). A flambagem de uma coluna pode resultar em uma falha abrupta de uma estrutura ou mecanismo e por isso há de se dedicar atenção para que as colunas não sofram esse fenômeno (HIBBELER, 2010).

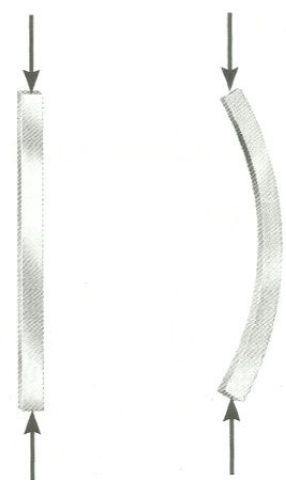

Figura 1. Flambagem Mecânica (HIBBELER, 2010)

Além disso, segundo Beer e Johnston (1995), quando componentes estruturais esbeltos são submetidos a uma variação de temperatura positiva, caracterizando um aumento de temperatura, seu material sofrerá uma dilatação térmica. Se o deslocamento axial da estrutura for restringido, surgirá então uma força interna de compressão que, dependendo de sua magnitude, poderá causar flambagem, sendo, nesse caso, chamada de flambagem térmica (Fig. 2).

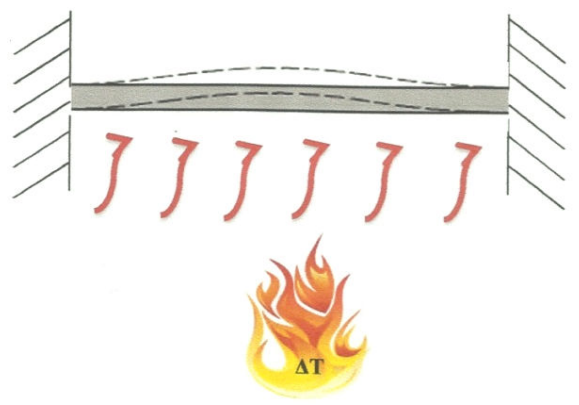

Figura 2. Flambagem Térmica

Observa-se que em várias situações torna-se necessário realizar perfurações nesses perfis de aço, como por exemplo, janela de visitas para observações e manutenções, passagem de cabos e tubulações, 
redução do próprio peso ou até mesmo somente por questões estéticas. Não existe nesses casos solução analítica para a determinação da variação da carga e da temperatura que poderiam causar a flambagem, o que reforça a importância do emprego da simulação numérica por permitir a condução de estudos que seriam de custo elevado ou até mesmo sem possibilidade de realização, caso fossem feitos experimentalmente.

Não se pode negligenciar o fato de que a remoção de material causa perdas na resistência mecânica do elemento estrutural. Neste sentido, desenvolveu-se um estudo de caso comparativo, removendo uma porcentagem do volume total do perfil, visando analisar a influência dessa remoção de material na variação da carga e temperatura críticas, com o objetivo de determinar a melhor geometria, baseado no método do Design Construtal, para o perfil, comum à Flambagem Térmica e à Flambagem Mecânica. Visando uma análise preliminar considerou-se apenas o componente estrutural birrotulado, com perfurações feitas ao longo da alma do perfil.

\section{SIMULAÇÃO NUMÉRICA}

Muitas vezes se torna complexo ou, até mesmo, inviável resolver problemas por métodos analíticos e experimentais, entre as diversas razões estão o custo elevado, a falta de equipamento e a própria impossibilidade de se resolver de maneira analítica e/ou experimental, em algumas situações.

Portanto, cada vez mais são empregadas soluções numéricas a partir da modelagem computacional, devido ao praticamente infinito número de possibilidades, ao custo relativamente baixo e à versatilidade das soluções numéricas se comparado aos demais métodos (SOUZA e BARBOZA, 2014).

Neste trabalho foi adotado o software ANSYS, para aplicações em análise estrutural, para determinar, numericamente, a variação crítica de temperatura e a carga critica, ou seja, a variação de temperatura que causa a flambagem térmica e a carga que causa flambagem mecânica em elementos estruturais esbeltos do tipo coluna. O software ANSYS é uma ferramenta computacional que usa como método de discretização o Método dos Elementos Finitos (MEF), permitindo análises estruturais lineares e não-lineares em problemas estáticos e dinâmicos, entre outras aplicações (ANSYS, 2005).

Em linhas gerais, o MEF é baseado na divisão do domínio de integração, contínuo, em um número finito de pequenas regiões denominadas elementos finitos, transformando o meio contínuo em discreto. $\mathrm{O}$ comportamento de cada elemento é arbitrado de forma aproximada, com a condição de que a malha formada pelos elementos se comporte de forma semelhante ao contínuo original. Na Fig. 3 é apresentado um exemplo que permite a visualização do conceito da divisão de um domínio contínuo em elementos.

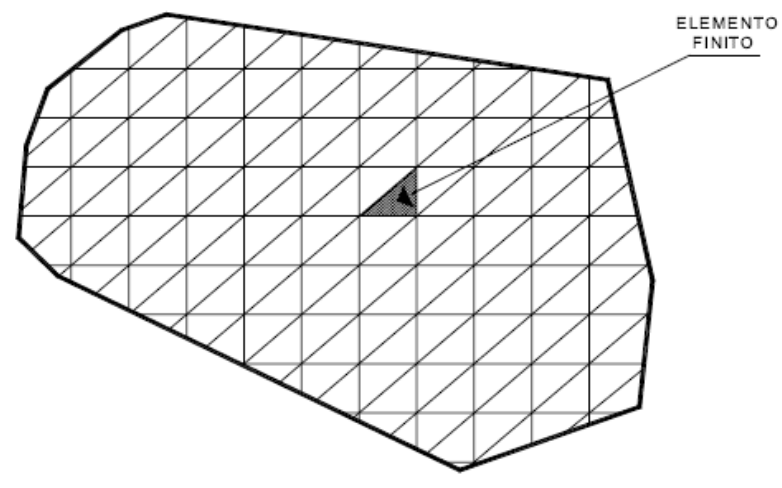

Figura 3. Divisão do domínio contínuo (VANALLI, 2004) 
Para o estudo numérico do fenômeno da flambagem térmica foi adotada a análise por autovaloresautovetores. As equações de equilíbrio por elementos finitos para este tipo de análise envolvem a solução de equações algébricas homogêneas cujo autovalor mais baixo corresponde à temperatura crítica de flambagem e o autovetor associado representa o primeiro modo de flambagem. Essa solução é análoga à determinação da carga crítica de flambagem mecânica em uma coluna, onde o autovalor mais baixo indica a carga crítica e o autovetor correspondente define o primeiro modo de flambagem (MADENCI e GUVEN, 2006).

Para o estudo de flambagem térmica, a diferença entre a temperatura de referência e a temperatura aplicada na coluna deve ser de apenas $1{ }^{\circ} \mathrm{C}$, pois os autovalores e autovetores são calculados para uma carga unitária aplicada. Assim, com essa diferença de $1^{\circ} \mathrm{C}$, o primeiro autovalor encontrado será igual à diferença de temperatura que causa a flambagem térmica (CASSENTI, 2012). No ANSYS o problema de autovaloresautovetores é resolvido usando o método numérico de Lanczos (ANSYS, 2005).

Segundo Anandrao et al. (2013), no caso de flambagem do elemento, com extremidades imóveis, sujeitos a um aumento de temperatura uniforme, acima da temperatura ambiente, o problema de autovalores é resolvido através da Eq. (1).

$$
[\mathrm{K}]\{\mathrm{U}\}-\lambda_{\mathrm{b}}\left[\mathrm{K}_{\mathrm{G}}\right]\{\mathrm{U}\}=0
$$

Onde: $[\mathrm{K}]$ é a matriz de rigidez elástica, $\left[\mathrm{K}_{\mathrm{G}}\right]$ é a matriz de rigidez geométrica, $\lambda_{\mathrm{b}}$ é o autovalor indicando a temperatura de flambagem e $\{\mathrm{U}\}$ é o autovetor (modo de flambagem).

E para o estudo de flambagem mecânica, a formulação utilizada na análise numérica inclui tanto os termos lineares como os não-lineares. Assim, a matriz de rigidez total [K], é obtida pela soma da matriz de rigidez convencional para pequenas deformações, $\left[\mathrm{K}_{\mathrm{E}}\right]$, com a matriz de rigidez geométrica, $\left[\mathrm{K}_{\mathrm{G}}\right]$. A matriz $\left[\mathrm{K}_{\mathrm{G}}\right]$ depende não só da geometria, mas também do esforço interno existente no início do carregamento, $\left\{\mathrm{P}_{0}\right\}$. Então, a matriz de rigidez total da coluna para um nível de carga $\left\{\mathrm{P}_{0}\right\}$ pode ser escrita como:

$$
[\mathrm{K}]=\left[\mathrm{K}_{\mathrm{E}}\right]+\left[\mathrm{K}_{\mathrm{G}}\right]
$$

Quando a carga atinge o nível de $\left\{\mathrm{P}_{0}\right\}=\lambda\left\{\mathrm{P}_{0}\right\}$, onde $\lambda$ é um escalar, a matriz de rigidez pode ser definida como:

$$
[\mathrm{K}]=\left[\mathrm{K}_{\mathrm{E}}\right]+\lambda\left[\mathrm{K}_{\mathrm{G}}\right]
$$

sendo $\lambda$ um escalar. As equações de equilíbrio governantes para a coluna podem ser escritas como:

$$
\left[\left[\mathrm{K}_{\mathrm{E}}\right]+\lambda\left[\mathrm{K}_{\mathrm{G}}\right]\right]\{\mathrm{U}\}=\lambda\left\{\mathrm{P}_{0}\right\}
$$

sendo $\{\mathrm{U}\}$ o vetor de deslocamento total, que pode, portanto, ser determinado por:

$$
\{\mathrm{U}\}=\left[\left[\mathrm{K}_{\mathrm{E}}\right]+\lambda\left[\mathrm{K}_{\mathrm{G}}\right]\right]^{-1} \lambda\left\{\mathrm{P}_{0}\right\}
$$

$\mathrm{Na}$ flambagem, o componente estrutural apresenta um grande crescimento nos deslocamentos sem crescimento da carga. Por definição matemática é possível determinar a matriz inversa como a matriz adjunta dividida pelo determinante dos coeficientes, então os deslocamentos $\{U\}$ tendem a infinito quando:

$$
\operatorname{det}\left[\left[\mathrm{K}_{\mathrm{E}}\right]+\lambda\left[\mathrm{K}_{\mathrm{G}}\right]\right]=0
$$

A Equação (6) representa um problema de autovalores, que quando resolvido gera o menor autovalor, que corresponde à carga crítica $\left\{\mathrm{P}_{\mathrm{cr}}\right\}=\lambda_{1}\left\{\mathrm{P}_{0}\right\}$ em que ocorre flambagem. Além disso, o vetor de deslocamento associado $\{\mathrm{U}\}$ define a forma do modo de flambagem. 


\section{DESIGN CONSTRUTAL}

Segundo Bejan e Lorente (2008), a aplicação da Lei Construtal é feita com o método Construtal. Este método é denominado Design Construtal e tem sido usado para predizer e explicar muitos fenômenos da natureza. Além disso, tem sido amplamente aplicado em problemas de engenharia, principalmente nas áreas de mecânica dos fluidos e de transferência de calor, para melhoria do seu desempenho.

Como exemplo de aplicações na natureza, da teoria Construtal, é possível citar: árvores, folhas, pulmões, escoamento de rios e raios. Existem também aplicações sociais como o fluxo de carros e o fluxo de pessoas na cidade. Observar-se algumas dessas aplicações na Fig. 4. E ainda as aplicações de engenharia (Design Construtal) como, por exemplo, resfriamento de circuitos eletrônicos, trocadores de calor em motores, energia das ondas, indústria de refrigeração e dimensionamento de estruturas.

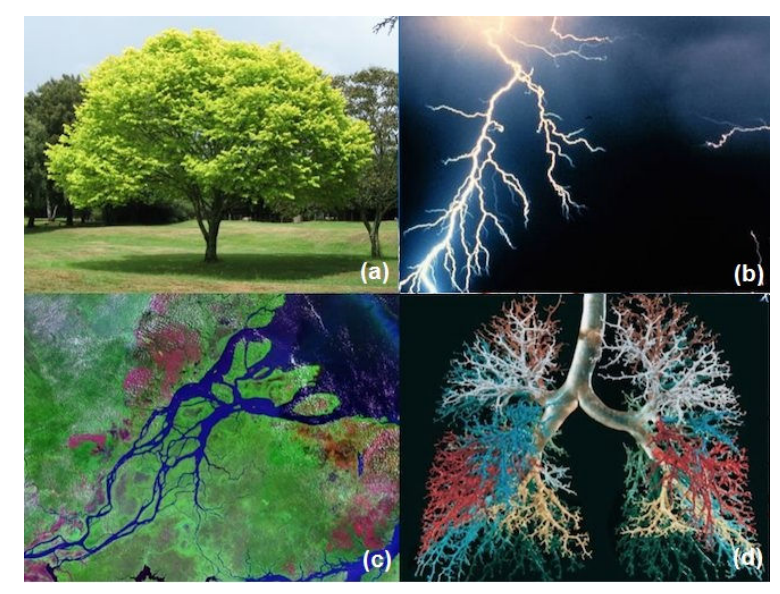

Figura 4. Exemplos de aplicações da Lei Construtal: (a) árvore, (b) escoamento de raios, (c) escoamento de rios e (d) pulmões. (Adaptado de http://www.timetobreakaway.com/blog/2015/6/9/freedom-leads-tostructure)

Esse princípio pode ser aplicado em sistemas de qualquer escala, desde a microscópica até a macroscópica, propiciando o desenvolvimento de estruturas complexas de escoamentos em engenharia e na natureza. Assim sendo, é possível verificar inúmeros exemplos de aplicação do referido princípio, como na Biologia, Geofísica e sistemas naturais nas quais as geometrias se modificam a fim de alcançar um objetivo que é, essencialmente, a minimização da resistência global para suas correntes internas (BEJAN, 2000).

Dessa forma, a Teoria Construtal conduz engenheiros à descoberta de arquiteturas de fluxo eficientes, quaisquer que sejam eles: fluidos, massa, energia e movimento em geral. Os projetos gerados são validados por comparações com a natureza, em sistemas animados e inanimados (RODRIGUES, 2014).

\section{METODOLOGIA}

Foi feita a verificação do modelo computacional, a partir da comparação da solução numérica com a solução teórica. Após verificação, o modelo foi utilizado para análise do comportamento da coluna perfurada. Uma determinada quantidade de material foi retirada da coluna. Para essa fração de volume, um tipo de furo foi considerado, o furo elíptico e diferentes configurações para o mesmo foram estudadas, variando as dimensões dos furos e estabelecendo uma relação entre elas, denominada de grau de liberdade.

Sendo assim, o estudo de caso foi realizado de acordo com o método Design Construtal. Possibilitando determinar a influência causada por perfurações na variação crítica de temperatura e na carga crítica. 
O componente estrutural estudado é do tipo coluna, feito de aço A-36 com módulo de elasticidade $\mathrm{E}$ $=210 \mathrm{GPa}$, coeficiente de Poisson $v=0,3$ e coeficiente de dilatação térmica $\alpha=12 \times 10^{-6}{ }^{\circ} \mathrm{C}^{-1}$, a partir de um perfil W310 $\times 21$. O componente (Fig. 5) possui as seguintes dimensões: comprimento $\mathrm{L}=4,5 \mathrm{~m}$, altura $\mathrm{d}=$ $303 \mathrm{~mm}$, espessura da aba $t=5,7 \mathrm{~mm}$, largura da aba $b=101 \mathrm{~mm}$ e espessura da alma $e=5,08 \mathrm{~mm}$. Possui ainda área de seção transversal $A=2680 \mathrm{~mm}^{2}$ e momentos de inércia $\mathrm{I}_{\mathrm{z}}=37 \times 10^{6} \mathrm{~mm}^{4}$ e $\mathrm{I}_{\mathrm{y}}=0,986 \times 10^{6}$ $\mathrm{mm}^{4}$.

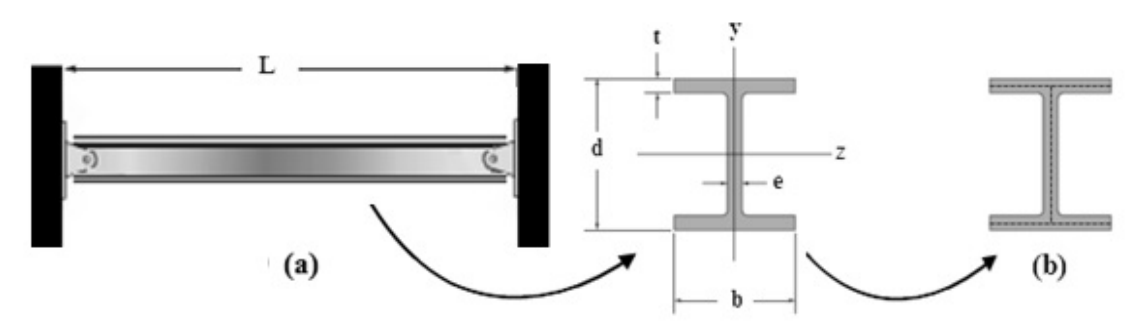

Figura 5. Componente estrutural: (a) Coluna bi-rotulada, formato e dimensões do perfil e (b) hipótese simplificativa

Para o modelo computacional usado no presente estudo foi adotado o elemento SHELL93, por ser um dos elementos do software ANSYS adequado para a simulação numérica de placas e cascas finas, permitindo que perfurações sejam consideradas ao longo da estrutura. $\mathrm{O}$ elemento SHELL93, apresentado na Fig. 6, possui oito nós e seis graus de liberdade por nó, ou seja, três translações nas direções $x, y$ e $z\left(u_{x}, u_{y}\right.$ e $\left.u_{z}\right)$ e três rotações em torno dos eixos $x, y$ e $z\left(r_{x}, r_{y}\right.$ e $\left.r_{z}\right)$, totalizando quarenta e oito graus de liberdade por elemento (ANSYS, 2005). Esse elemento finito é definido pelo modelo de uma superfície e sua espessura é atribuída durante o processo de modelagem computacional.

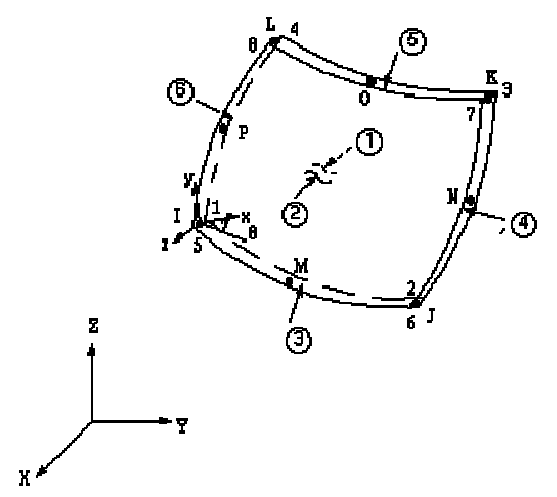

Figura 6. Elemento SHELL93 (Ansys User's Manual, 2005)

\subsection{Verificação do modelo computacional}

As verificações dos modelos computacionais foram realizadas, comparando o valor da variação crítica de temperatura com a solução analítica dada pela Eq. (7) (Hibbeler, 2010), para o estudo da flambagem térmica.

$$
\Delta T_{c r}=\frac{\pi^{2} I}{\alpha A L^{2}}
$$


E para a flambagem mecânica, comparando o valor da carga crítica com a solução analítica dada pela Eq. (8) (Hibbeler, 2010).

$$
P_{e r}=\frac{\pi^{2} E l}{L^{2}}
$$

A variação crítica de temperatura obtida numericamente foi de $\Delta T_{c r}=15,18^{\circ} \mathrm{C}$, que apresentou uma diferença de $1,61 \%$ em relação à variação crítica de temperatura definida pela Eq. (7), que foi de $\Delta T_{c r}=$ $14,94{ }^{\circ} \mathrm{C}$. E a carga crítica obtida numericamente foi de $P_{s r}=105,15 \mathrm{kN}$ e carga Crítica analítica, definida pela Eq. (8), foi de $P_{\mathrm{crr}}^{*}=100,51 \mathrm{kN}$, resultando uma diferença de $4,6 \%$.

Considerando as diferenças entre as soluções numéricas e analíticas, é possível afirmar que os modelos computacionais foram verificados.

\subsection{Estudo de caso}

Foi feito um estudo de caso removendo $15 \%$ do volume total da coluna apresentada na Fig. 5, visando analisar a influência dessa remoção de material na variação crítica de temperatura da coluna e na carga crítica. Para todos os casos avaliados foi considerado um componente estrutural birrotulado, com perfurações feitas ao longo da alma da coluna. Como a condição de vinculação é birrotulada, considerou-se para ambas extremidades as seguintes condições de contorno: nas linhas médias das abas $u_{y}=r_{x}=r_{z}=0 \mathrm{e}$ nas linhas médias das almas $u_{x}=u_{y}=u_{z}=r_{x}=r_{z}=0$.

Para a discretização da coluna foram adotados elementos finitos triangulares, pois estes se adaptam melhor às geometrias complexas como as formadas com a inclusão das perfurações. Baseando-se em estudos anteriores, gerou-se a malha com elementos finitos de tamanho $20 \mathrm{~mm}$.

Para a determinação do número de furos necessários para alcançar 15\% do material, usou-se a seguinte equação:

$$
\phi=\frac{V_{0}}{V}
$$

onde: $V_{0}$ é o volume do material a ser removido e $V$ o volume total da coluna.

Então, usando $\square=0,15$, a partir da Eq. (9), definiu-se o volume de material que será removido. Sabendo que as perfurações serão feitas somente na alma, é possível determinar a área total que deverá ser removida. É importante observar que a espessura da alma é diferente das espessuras das abas. E está primeira que deverá ser utilizada nos cálculos.

Tem-se, então, os seguintes valores: $V_{0}=1777091,40 \mathrm{~mm}^{3}$ (volume a ser removido) e $A_{0}=349821,1417 \mathrm{~mm}^{2}$ (área a ser removida).

Para determinar o número de perfurações levou-se em consideração a altura da alma (menor dimensão, ver Fig. 5) e utilizou-se o princípio de Saint-Venant para definir a distância mínima das extremidades a partir da qual poderiam ser realizadas as perfurações. Além disso, as perfurações foram realizadas equidistantes, permitindo a realização de quatorze furos. Logo: $A_{f u r o}=24987,22 \mathrm{~mm}^{2}$ (área de cada furo).

Foram estudados furos do tipo elíptico. O número de furos e a área de cada furo foram mantidos constantes, ou seja, $\square=0,15$ distribuídos em quatorze perfurações. Para avaliar a influência da forma do furo, foi empregado o grau de liberdade $H_{0} / L_{0}$, que relaciona as dimensões características $H_{0}$ e $L_{0}$ das perfurações, indicadas na Fig. 7. 


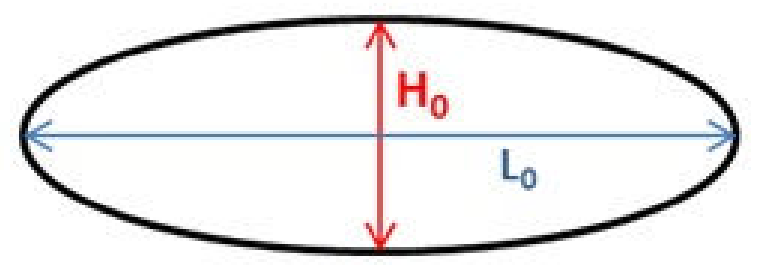

Figura 7. Indicação do $H_{0}$ e $L_{0}$ da perfuração Elíptica

\section{RESULTADOS E DISCUSSÃO}

Foram feitos estudos do comportamento das estruturas perfuradas submetidas à flambagem térmica $\mathrm{e}$ à flambagem mecânica. Foram feitas perfurações elípticas, diminuindo a cada caso o valor de $\mathrm{L}_{0} \mathrm{e}$ consequentemente aumentando o valor de $\mathrm{H}_{0}$, para manter sempre a mesma área da perfuração. Os casos estudados estão apresentados na Tab. 1, com seus respectivos valores para a variação crítica de temperatura e carga crítica. Além disso, esses valores para a $\Delta T_{\text {cr }}$ foram divididos pela variação crítica de temperatura da coluna sem perfuração, definindo assim a variação crítica de temperatura adimensional $\Delta T_{\text {cr,adm. }}$ O mesmo foi feito com a carga crítica, dividiu-se o valor de $\mathrm{P}_{\text {cr }}$, obtidos para esses casos, pelo valor da carga crítica da coluna sem perfuração, definindo assim a carga crítica adimensional, $\mathrm{P}_{\mathrm{cr}, \mathrm{adm}}$.

Tabela 1. Valores obtidos para a variação crítica de temperatura e carga crítica (Perfil com perfuração elíptica)

\begin{tabular}{cccccccc}
\hline Caso & $\left.\mathbf{L}_{\mathbf{0}} \mathbf{( m m}\right)$ & $\mathbf{H}_{\mathbf{0}}(\mathbf{m m})$ & $\mathbf{H}_{\mathbf{0}} / \mathbf{L}_{\mathbf{0}}$ & $\boldsymbol{\Delta} \boldsymbol{T}_{\mathbf{c r}}\left({ }^{\mathbf{o}} \mathbf{C}\right)$ & $\boldsymbol{\Delta} \boldsymbol{T}_{\text {cr,adm }}$ & $\boldsymbol{P}_{\text {cr }}(\mathbf{K N})$ & $\boldsymbol{P}_{\text {cr,adm }}$ \\
\hline 1 & 250,00 & 127,26 & 0,51 & 18,762 & 1,24 & 104,892 & 0,9975 \\
2 & 236,00 & 134,81 & 0,57 & 19,023 & 1,25 & 104,933 & 0,9979 \\
3 & 222,00 & 143,31 & 0,65 & 19,332 & 1,27 & 104,959 & 0,9982 \\
4 & 208,00 & 152,96 & 0,74 & 19,704 & 1,30 & 104,977 & 0,9983 \\
5 & 194,00 & 163,99 & 0,85 & 20,162 & 1,33 & 104,992 & 0,9985 \\
6 & 178,37 & 178,37 & 1,00 & 20,817 & 1,37 & 104,999 & 0,9985 \\
7 & 163,99 & 194,00 & 1,18 & 21,614 & 1,42 & 105,004 & 0,9986 \\
8 & 152,96 & 208,00 & 1,36 & 22,404 & 1,48 & 105,005 & 0,9986 \\
9 & 143,31 & 222,00 & 1,55 & 23,284 & 1,53 & 105,006 & 0,9986 \\
10 & 134,81 & 236,00 & 1,75 & 24,239 & 1,60 & 105,005 & 0,9986 \\
11 & 127,26 & 250,00 & 1,96 & 25,293 & 1,67 & 105,006 & 0,9986 \\
\hline
\end{tabular}

Com a variação de $H_{0} / L_{0}$, foram obtidas perfurações com forma de elipse, alongadas horizontalmente $\left(H_{0} / L_{0}<1\right)$ ou verticalmente $\left(H_{0} / L_{0}>1\right)$, e uma perfuração circular $\left(H_{0} / L 0=1,00\right)$. Analisando a Tab. 1 é possível perceber que quanto maior for a relação $H_{0} / L_{0}$ maior é o valor da $\Delta T_{c r, a d m}$, porém essa tendência não ocorre em sua totalidade para os valores de $\mathrm{P}_{\text {cr,adm, }}$, isso fica evidente no gráfico da Fig. 8, que apresenta a carga crítica e a variação crítica de temperatura adimensionais em função da relação $\mathrm{H}_{0} / \mathrm{L}_{0}$. 


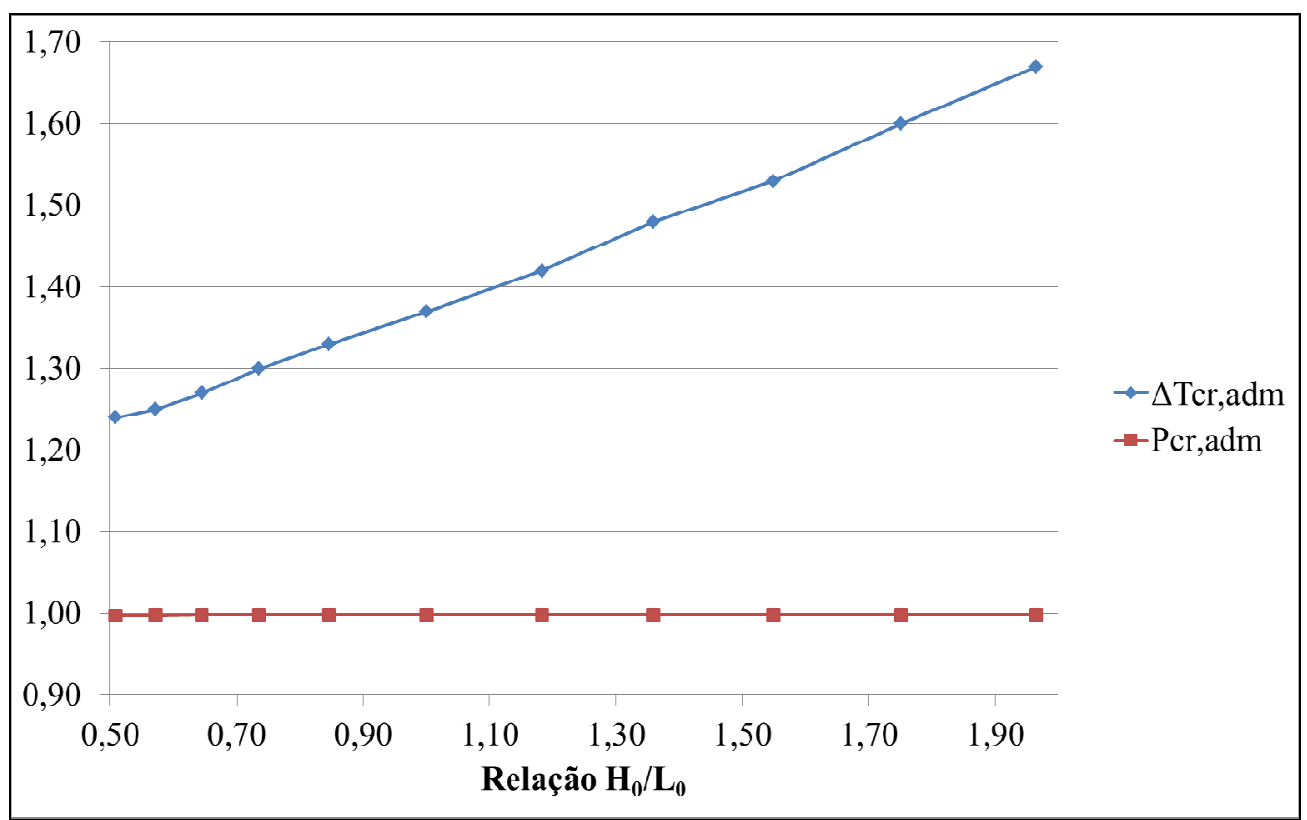

Figura 8. Variação crítica de temperatura e carga crítica para diferentes perfurações elípticas

\section{CONCLUSÕES}

Nesse trabalho foram desenvolvidos e verificados modelos computacionais capazes de solucionar os problemas da flambagem térmica e mecânica em componentes estruturais esbeltos (colunas), birrotulados. A partir desses modelos computacionais foi possível estudar situações práticas que não possuem solução analítica, que é a influência de perfurações, distribuídas ao longo do componente estrutural, na flambagem térmica e mecânica. Empregando o método Constructal Design, foram analisados e comparados, os resultados para perfurações elípticas. Estudou-se a influência da variação da forma da perfuração na flambagem térmica e mecânica, mantendo-se fixo o volume de material retirado da coluna.

Para o estudo da flambagem térmica, todos valores para $\Delta \mathrm{T}_{\text {cr,adm }}$ são maiores que 1 , ou seja, todos os valores de $\Delta \mathrm{T}_{\mathrm{cr}}$ das colunas perfuradas são maiores que a $\Delta \mathrm{T} c r$ da coluna sem perfuração. $\mathrm{E}$ quanto maior $\mathrm{H}_{0} / \mathrm{L}_{0}$ maior a $\Delta \mathrm{T}_{\mathrm{cr}}$. O maior e o menor valor, para a variação de temperatura crítica, encontrados foram os valores relacionados com o maior e o menor grau de liberdade respectivamente. Isso não ocorreu em sua totalidade para os valores de $\mathrm{P}_{\mathrm{cr}, \mathrm{adm}}$, muito provavelmente em virtude das furações terem sido efetuadas alinhadas com o eixo neutro do perfil.

Pode-se observar, também que a variação obtida na carga crítica adimensional não é significativa na Flambagem Mecânica, pois ela ocorre na terceira casa decimal. Sendo assim, considerando flambagem térmica e flambagem mecânica, a melhor geometria é a que apresenta maior valor para a relação $\mathrm{H}_{0} / \mathrm{L}_{0}\left(\mathrm{H}_{0} / \mathrm{L}_{0}=1,96\right)$, pois para a flambagem térmica ela promove um aumento significativo no valor da variação crítica de temperatura. Em outras palavras, quem está determinando a geometria ótima, neste caso, é a análise da flambagem térmica e não a análise da flambagem mecânica.

Os resultados obtidos mostraram que o método Design Constructal permite a determinação de geometrias otimizadas que conduzem a performances superiores dos componentes estruturais analisados. Outras sugestões para trabalhos futuros, seriam: estudos envolvendo diferentes tipos de perfis para a coluna, diferentes tipos de perfurações (por exemplo, furos retangulares, hexagonais e oblongos), diferentes vinculações e diferentes valores para a fração volumétrica das perfurações. 


\section{REFERÊNCIAS BIBLIOGRÁFICAS}

ANANDRAO, K. S. et al. Thermal Buckling and Free Vibration Analysis of Heated Functionally Graded Material Beams. Defence Science Journal, vol. 63, n. 3, pp. 315-322, 2013.

ANSYS. User's Manual (version 10.0). Swanson Analysis System Inc, 2005.

BEER, F. P., JOHNSTON, E. R. Jr. Resistência dos Materiais. Makron Books, 1995.

BEJAN, A. Shape and Structure, from engineering to nature, Cambridge University Press, 2000.

BEJAN, A. AND LORENTE, S. Design with Constructal Theory. Wiley, Hoboken, 2008.

CASSENTI, B. Thermal Buckling of a 2D Beam Fixed at Both Ends. University of Connecticut, , 2012. Disponível em: http://www.engr.uconn.edu/ cassenti/

HIBBELER, R. C. Resistência dos Materiais. Pearson Prentice Hall, 2010.

MADENCI, E. E GUVEN, I. The Finite Element Method and Applications in Engineering Using ANSYS®. Springer, 2006.

RODRIGUES, M. K. Modelagem computacional aplicada à melhoria do desempenho térmico de trocador de calor solo-ar através do método Constructal Design. Dissertação de mestrado em Modelagem Computacional, Universidade Federal do Rio Grande, 2014.

SOUZA, C. M. E BARBOZA, V. O. Simulação numérica aplicada à melhoria do comportamento mecânico de tubos estruturais perfurados submetidos à flambagem elástica. Trabalho de conclusão de curso de Engenharia Mecânica, Universidade Federal do Rio Grande, 2014.

VANALLI, L. O MEC e o MEF aplicados à análise de problemas viscoplásticos em meios anisotrópicos e composto. Tese de doutorado em Engenharia de Estruturas, Universidade São Paulo, 2004. 